Sociologie et sociétés

\title{
Femmes et syndicats en France
}

Women and Unions in France

Mujeres y sindicatos en Francia

\section{Madeleine GUILBERT}

Volume 6, numéro 1, mai 1974

Femme, travail, syndicalisme

URI : https://id.erudit.org/iderudit/001318ar

DOI : https://doi.org/10.7202/001318ar

Aller au sommaire du numéro

Éditeur(s)

Les Presses de l'Université de Montréal

ISSN

0038-030X (imprimé)

1492-1375 (numérique)

Découvrir la revue

Citer cet article

GUILBERT, M. (1974). Femmes et syndicats en France. Sociologie et sociétés, 6(1), 157-170. https://doi.org/10.7202/001318ar

\section{Résumé de l'article}

Pour aborder les problèmes des rapports entre les femmes et les syndicats en France il faut d'abord replacer les faits dans leur contexte historique. La montée de la main-d'œuvre féminine, mise en situation de concurrence avec la main-d'œuvre masculine, a suscité, dès la seconde moitié du 19 siècle, dans le mouvement syndical, des inquiétudes et des oppositions qui n'ont été surmontées que lentement. Si on examine les faits actuels, il faut tenir compte d'une situation particulière des femmes, sur le plan familial comme sur le plan du travail. Les taux d'activité des femmes sont, depuis plusieurs années, en nette augmentation mais la répartition des femmes actives, notamment suivant la catégorie socio-professionnelle ou la qualification, est différente de celle des hommes. Ces faits peuvent avoir des conséquences négatives. En même temps cependant l'attention actuellement portée aux problèmes des conditions de travail ne peut manquer d'être favorable au développement de la participation des femmes aux luttes ouvrières. La pluralité syndicale en France rend difficile une appréciation globale de l'état actuel de la participation syndicale des femmes. Importante à la base, celle-ci est moins importante à mesure que l'on s'élève dans l'échelle des responsabilités. Il est certain toutefois que la période actuelle est marquée par une accentuation de la participation et de l'initiative des femmes dans les actions syndicales.
Tous droits réservés @ Les Presses de l'Université de Montréal, 1974
Ce document est protégé par la loi sur le droit d'auteur. L'utilisation des services d'Érudit (y compris la reproduction) est assujettie à sa politique d'utilisation que vous pouvez consulter en ligne.

https://apropos.erudit.org/fr/usagers/politique-dutilisation/ 


\section{Femmes et syndicats en France}

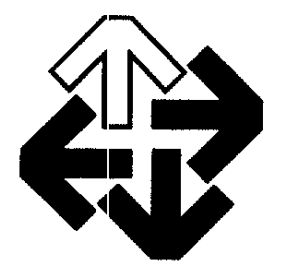

MADELEINE GUILBERT

IL NE SEMBLE GUÈRE POSSIBLE d'aborder les problèmes des rapports entre les femmes actives et l'organisation syndicale dans leurs aspects actuels sans envisager, même rapidement, ces problèmes dans leur contexte historique. Ceci est sans doute vrai pour tous les pays. On peut se demander toutefois si, dans le cas de la France où l'histoire du mouvement syndical est longue et complexe, où le travail des femmes a suscité très tôt des préoccupations, la nécessité d'un bref rappel des faits antérieurs ne s'impose pas particulièrement. La situation actuelle de la main-d'œuvre féminine, sur le plan syndical, y est l'aboutissement d'une lente évolution dont il est indispensable de retracer les étapes et de situer les points clés.

La reconnaissance officielle des syndicats en France remonte à 1884. La période qui sépare cette date de celle du début de la Première Guerre mondiale voit le développement d'un mouvement syndical dominé par la crainte du chômage et par le souci de la défense des tarifs; mouvement fortement marqué par la prédominance, dans de nombreuses fédérations, des dirigeants anarcho-syndicalistes et en même temps par l'influence des idéologies proudhoniennes; mouvement qui se caractérise par l'importance de ses luttes plutôt que par des préoccupations concernant celle de ses effectifs.

Dès avant la seconde moitié du $19^{\mathrm{e}}$ siècle, lors des premiers développements de la grande industrie, on avait assisté à une montée considérable du nom- 
bre et du pourcentage de femmes dans la population active. Occupées auparavant à la production textile familiale qui s'était étendue dans les campagnes au cours du $18^{\mathrm{e}}$ siècle, lors du déclin des corporations, elles ont été appelées en masse d'abord dans les fabriques de l'industrie textile ${ }^{1}$. Elles entreront dans les autres branches à mesure que celles-ci se développeront. Le nombre des femmes occupées dans les industries de transformation est déjà de 1269700 en 1866; il passe à 2254300 en 1906. Elles pénétreront plus tard dans les emplois du commerce et des banques où elles seront 771000 en 1906.

Le développement de l'emploi féminin dans les professions industrielles au cours de la seconde moitié du 19 siècle est, pour le mouvement syndical, une source d'inquiétudes. Si les femmes entrent en grand nombre dans les entreprises c'est en effet surtout parce qu'on peut leur faire accepter des salaires très bas (de 40 à $50 \%$ inférieurs à ceux des hommes d'après les enquêtes réalisées à l'époque par l'Office du travail ${ }^{2}$ ). Dans de nombreux cas même, des ouvrières embauchées à bas prix remplacent des ouvriers. Cette utilisation de la maind'œuvre féminine, ainsi mise en concurrence avec la main-d'œuvre masculine, risque de peser sur le niveau général des salaires. Une telle situation est sans doute d'autant plus durement ressentie dans la classe ouvrière que le travail des femmes hors du foyer est alors, dans sa forme massive, un fait relativement nouveau qui frappe les esprits et heurte les conceptions traditionnelles.

Certes, dès 1848, Marx affirmait que, malgré les misères engendrées, le travail des femmes est une occasion de libération puisqu'il les amène inéluctablement à participer, aux côtés des hommes, aux luttes de la classe ouvrière. Mais les idées de Marx ont alors peu pénétré le mouvement ouvrier français et, pour donner à leurs craintes des justifications idéologiques, c'est le plus souvent à Proudhon que se réfèrent ceux des syndicalistes qui demandent le retour de la femme au foyer d'où, selon eux, elle n'aurait jamais dû sortir ${ }^{3}$.

La période qui s'étend jusqu'à la Première Guerre mondiale est, en définitive, marquée par l'inquiétude des syndicats devant la montée de l'emploi féminin et caractérisée par des attitudes diverses suivant les secteurs, suivant l'idéologie dominante dans chacun d'eux, suivant l'importance qu'y revêt la concurrence féminine : opposition au travail des femmes et à leur admission au syndicat dans la Fédération du livre, par exemple; attitudes plus nuancées, tentatives souvent teintées de paternalisme pour les syndiqués dans d'autres secteurs comme l'habillement, l'alimentation. La question du travail des femmes est à

1. La célèbre enquête faite par le $\mathrm{D}^{\mathrm{r}}$ Villermé, à la demande de l'Académie des sciences morales et politiques a été réalisée entre 1837 et 1839 dans les principaux centres de l'industrie textile française. Elle montre bien que, dès cette époque, alors que l'industrie textile familiale subsiste encore en partie dans les campagnes, de nombreuses femmes - et avec elles de nombreux enfants - travaillent déjà dans les fabriques, surtout dans les filatures. Villermé, Tableau de l'état physique et moral des ouvriers employés dans les manufactures de coton, de laine et de soie, 2 vol., Paris, Renouard, 1840, partiellement réédité à Paris par l'Union générale d'édition, «10 × $18 », 1971$.

2. Bordereaux de salaires pour diverses catégories d'ouvriers en 1900 et 1901, publication de l'Office du travail, Paris, Imprimerie nationale, 1902, $233 \mathrm{p}$.

3. On sait que Proudhon, dont l'influence fut considérable lors des premiers développements du syndicalisme en France, a exprimé à de nombreuses reprises l'idée que la femme est avant tout mère, épouse et ménagère, que là est sa vocation profonde, que le travail salarié hors du foyer est contraire à sa véritable nature (voir surtout la Pornocratie ou les femmes dans les temps modernes, Paris, A. Lacroix, 1875 et De la justice dans la révolution et dans l'Église, $\mathrm{t}$. IV, $10^{\mathrm{e}}$ et $11^{\mathrm{e}}$ étude : Amour et mariage, Bruxelles, Lacroix, Verboeckhoven et $C^{\mathrm{ie}}, 1869$ ). 
l'ordre du jour ou fait l'objet d'interventions importantes dans neuf des douze congrès de la C.G.T. (Confédération générale du travail, née en 1895) qui eurent lieu entre 1895 et 1914 mais la représentation féminine y est quasi nulle ${ }^{4}$.

À la fin de cette période, cependant, à la veille de 1914, on voit s'amorcer une rnutation. À quoi faut-il l'attribuer? D'abord, sans aucun doute, à une participation importante des femmes aux grandes grèves qui ont marqué l'histoire syndicale jusqu'en 1914 (grèves de 1893, 1900, 1902, 1904, 1906, 1910, 1912). On observe même des grèves de femmes dont la durée et l'âpreté sont soulignées dans la presse ouvrière : grève des casseuses de sucre en 1892, des corsetières de Limoges en 1895 (108 jours), des cartonnières de La Guerche en 1901 (130 jours), des sardinières de Douamenez en 1905, des ouvrières en soie de Vizille en 1905 ( 115 jours), des fileuses des Cévennes en 1906, des fromagères de Roquefort en 1907, des confectionneuses de la maison Esders en 1911, des ouvrières de la sucrerie Lebaudy en 1913, etc. On fait remarquer, certes, que l'affiliation syndicale des femmes, en forte croissance au cours de ces mouvements, décrcît rapidement dans les périodes qui suivent. Il n'en reste pas moins que, si on compare les chiffres de 1914 à ceux de 1900, le nombre de femmes syndiquées a proportionnellement augmenté davantage que celui des hommes ${ }^{5}$ et que le ton de la presse syndicale à leur égard a changé progressivement ${ }^{6}$.

La seconde raison du changement qui s'amorce avant 1914 pourrait apparaître comme purement anecdotique. C'est l'affaire Couriau au cours de laquelle le Syndicat lyonnais du livre, passant outre aux décisions du congrès fédéral de 1910 , refuse l'adhésion d'une femme typographe ${ }^{7}$. Il n'en reste pas moins que si, à la suite de l'inquiétude que suscite la tentative de «récupération » de l'incident par le mouvement féministe ${ }^{8}$, un courant de réflexion s'établit, dans la C.G.T., sur la nécessité d'une action cohérente pour élargir la participation syndicale des femmes, ce courant de réflexion n'aurait pu avoir lieu sans une évolution préalable des esprits. Il est clairement dit, dès ce moment, que les oppositions

4. Trois femmes déléguées au Congrès de Limoges en 1895, deux à Paris en 1900, seize à Lyon en 1901 (toutes habitant à Lyon), deux à Montpellier en 1902, une à Amiens en 1906, deux à Marseille en 1908, trois à Toulouse en 1910. Voir M. Guilbert, les Femmes et l'organisation syndicale avant 1914, Paris, Éditions du C.N.R.S., 1966, deuxième partie : "Les congrès", p. 41-201. 5. L'Annuaire des syndicats professionnels, publié chaque année, à partir de 1890, par le ministère du Commerce et de l'Industrie, donne, d'après les déclarations des syndicats, le nombre d'adhérents à chacun d'eux. $\dot{A}$ partir de 1900 il donne le nombre de femmes. Pour les syndiciats ouvriers, le nombre total d'adhérents passe, d'après l'Annuaire, de 588832 en 1900 à 1026302 en 1914. Le nombre de femmes parmi ces adhérents passe de $30975(5,26 \%$ en 1900) à $89364(8,7 \%$ en 1914). Il ne faut pas oublier cependant que le pourcentage de femmes dans la population active est de $36,7 \%$ en 1911 .

5. Pour l'analyse des articles de la presse syndicale de l'époque, voir M. Guilbert, op. cit., $4^{e}$ partie, p. $245-429$.

7. Emma Couriau, femme typographe, payée au tarif syndical, mariée à un typographe, voit refuser son adhésion par le Syndicat lyonnais du livre. Son mari est radié du syndicat. La section de Lyon invoque comme justification une décision d'assemblée genérale qui interdit à tout syndiqué uni à une "typote» de laisser exercer à celle-ci la typographie. L'affaire est d'abord prise en main par la Fédération féministe du Sud-Est. Elle suscite ensuite, à partir d'août 1913. dans la Vie ouvrière, la Bataille syndicaliste, et la Voix du peuple, organe officiel de la C.G.T., une série d'articles qui sont autant d'efforts de réflexion et de mise au point concernant l'attitude de la C.G.T. vis-à-vis des femmes (voir M. Guilbert, op. cit., p. 410 et suivantes).

3. Dans une récente thèse de doctorat de $3^{\mathrm{e}}$ cycle : Mouvement féministe et syndicalisme avant 1914, (378 p., document dactylographié), Marie-Hélène Zylberberg-Hocquard analyse les rapports entre le mouvement féministe et les syndicats et montre le rôle important joué, lors de l'affaire Couriau par les institutrices de la Fédération féministe universitaire parmi lesquelles Marie Guillot, auteur des principaux articles cités dans la note précédente. 
entre main-d'œuvre féminine et masculine ne sont qu'apparentes et que leurs intérêts sont fondamentalement communs.

Est-ce à dire que le mouvement syndical a désormais surmonté les difficultés que suscite la présence des femmes parmi les travailleurs? La période suivante, celle qui s'étend jusqu'à la Seconde Guerre mondiale est marquée, non par une progression continue, mais par des «bonds» successifs de la participation syndicale de la main-d'œuvre féminine. Fait remarquable tous les moments «forts» des luttes syndicales, au cours de cette période, sont marqués par une accentuation de l'influence des syndicats parmi les femmes et par l'accès progressif de celles-ci à des responsabilités de plus en plus importantes. Au cours des grandes grèves ouvrières qui marquent la fin de la guerre de 1914-1918, elles jouent un rôle décisif. En 1921, pour la première fois, une femme se voit élire à un poste de secrétaire fédérale (à la fédération C.G.T.U. ${ }^{9}$ de l'alimentation). En 1936, la participation des femmes aux grèves et aux occupations des locaux de travail est extrêmement importante (la grève des grands magasins dont le personnel est en majorité féminin a été l'un des épisodes marquants de ces grèves) et l'on voit augmenter considérablement le nombre des femmes responsables des syndicats de base. En 1945, le congrès de la C.G.T. confie, pour la première fois, à une femme un poste de secrétaire confédérale. L'évolution a été à la fois considérable et lente : considérable parce qu'elle a consacré des idées qui commençaient à peine à se faire jour à la veille de 1914. Lente parce que les progrès accomplis, bien qu'ils aient une signification exemplaire, portent sur un nombre restreint de cas. Elle nous fait pressentir l'importance du chemin parcouru au cours des dernières années depuis la fin de la Seconde Guerre mondiale. À cet égard, une date relativement récente, celle de 1968, marque dans cette progression une étape dont on ne saurait trop souligner l'importance. La participation des femmes aux grandes grèves ouvrières de mai et juin 1968 a été telle qu'on a pu dire qu'elles n'auraient pu avoir lieu sans cette adhésion massive des femmes. Nous retrouvons ici une idée exprimée plus haut, celle d'une concordance entre les périodes de luttes syndicales intenses et les périodes de montée de la participation syndicale des femmes.

II nous faut maintenant tenter d'analyser la situation actuelle, pour en saisir à la fois les aspects positifs et les difficultés, pour en appréhender la complexité. Nous devons examiner d'abord une série de faits concernant l'emploi féminin en France. Les taux d'activité féminins sont inférieurs aux taux d'activité masculins. Si l'exercice d'une profession est, depuis de longues années déjà, la règle générale pour les hommes en âge et en état de travailler, il n'en est pas de même pour les femmes. On a observé cependant, au cours des dernières années et contrairement à un mouvement de baisse globale des taux d'activité ${ }^{10}$, une tendance à l'augmentation des taux d'activité féminins. Cette augmentation est constatée plus particulièrement pour les tranches d'âge de 25 à 45 ans dans lesquelles les taux d'activité des femmes sont traditionnellement les plus faibles. Elle est particulièrement nette pour les femmes mariées. Il y a là une indication

9. Confédération générale du travail unitaire. Elle est née, en 1921, d'une scission à l'intérieur de la C.G.T. La réunification devait avoir lieu en 1936.

10. Cette baisse globale des taux d'activité est liée essentiellement à l'élévation de l'âge de la fin de la scolarité obligatoire et à l'abaissement de l'âge de la retraite pour certaines catégories. 
importante qui tend à montrer que, pour des raisons complexes qu'il faudrait atteindre au moyen d'enquêtes, l'accès des femmes à des occupations professionnelles en vient de plus à être considéré comme normal, même lorsqu'elles ont par ailleurs des charges familiales. Le pourcentage de femmes dans la population active française était évalué en 1972 à $38,2 \%$.

Est-ce à dire qu'il en est forcément de même lorsqu'il s'agit d'accéder à des préoccupations voire à des responsabilités d'ordre professionnel? Il est plus difficile de mesurer, dans ce domaine, une évolution qu'on ne saurait toutefois nier. Il est certain que des obstacles d'ordre psychologique se manifestent, souvent encore, pour freiner une évasion vers des préoccupations différentes de celles qui sont traditionnellement celles des femmes, même si elles exercent une profession. On a, jusque-là, beaucoup insisté sur ces résistances que l'on peut rencontrer chez les femmes elles-mêmes, dans le milieu familial et jusque dans le milieu de travail. Ce que nous dirons plus loin au sujet des activités syndicales des femmes dans la période actuelle laisse à penser cependant que, là aussi, les images et les attitudes traditionnelles tendent à s'estomper.

Il n'en reste pas moins un fait concret, sur lequel il faut insister : les femmes disposent, en moyenne, de beaucoup moins de temps que les hommes en dehors de leur travail professionnel. Des études de budgets-temps ${ }^{11}$ ont montré que celles qui sont mariées et ont des enfants assument encore une part considérable des occupations ménagères et familiales. Ces obstacles matériels sont réels et leur impact sur le comportement syndical des femmes est sans doute plus important qu'on ne pense généralement. On pourrait dire, pour résumer cette première série d'observations, que l'opposition entre le rôle traditionnel des femmes sur le plan familial et le fait d'assumer pleinement leur condition de travailleurs ne s'exprime plus guère au niveau des principes mais qu'elle est encore présente dans la vie quotidienne. Nous en aurons plus loin des échos à travers les témoignages des organisations syndicales.

Une deuxième série d'observations portera sur la situation professionnelle des femmes. Celle-ci présente en effet, à bien des égards, des aspects spécifiques : la répartition des femmes entre les diverses branches de l'emploi est différente de celle des hommes. En conséquence, le pourcentage de femmes dans la population active varie d'une branche à une autre. Lors du recensement de $1968^{12}$ on trouvait $29,9 \%$ de femmes dans les industries chimiques, $17,6 \%$ dans les industries des métaux, 52,9\% dans les industries textiles, $80,8 \%$ dans le travail des étoffes, 43,9\% dans les emplois du commerce et des banques, $41,9 \%$ dans les services publics et administratifs, 94,9\% dans les services domestiques. Encore ces distinctions devraient-elles être affinées. Dans les industries de métaux, par exemple, on trouve $35 \%$ de femmes dans les constructions électriques et le pourcentage est encore beaucoup plus élevé si l'on considère la seule fabrication des lampes électriques. On observe donc, d'une manière générale, - et ceci ne peut manquer de soulever des problèmes de tous ordres - l'existence

11. M. Guilbert, M. Lowit et J. Creusen, «Problèmes de méthode pour une enquête de budgets-temps", Revue française de sociologie, juillet-septembre 1965; "Enquête comparative de budgets-temps», Revue française de sociologie, octobre-décembre 1965; "Les budgets-temps et les horaires de la vie quotidienne", Revue française de sociologie, avril-juin 1967.

12. Le recensement de 1968 est le dernier en date. On a pu disposer depuis d'estimations. Le prochain recensement aura lieu en 1975. 
de secteurs à forte majorité féminine à côté de secteurs où les femmes sont peu représentées.

La répartition des hommes et des femmes suivant la catégorie socio-professionnelle est également différente. À titre d'exemple, signalons que le recensement de 1968 distinguait $0,8 \%$ de cadres administratifs supérieurs parmi les femmes actives contre $1,4 \%$ parmi les hommes ; $0,8 \%$ de techniciens parmi les femmes contre 3,6\% parmi les hommes; $20,8 \%$ d'employées de bureau parmi les femmes contre $6,9 \%$ parmi les hommes; $22 \%$ d'ouvrières parmi les femmes contre $45,8 \%$ d'ouvriers parmi les hommes.

Quant au statut dans la profession, on peut constater que le pourcentage de salariés était en 1968 de $75,4 \%$ parmi la population active masculine contre $72,6 \%$ parmi la population active féminine. On a observé toutefois, entre le recensement de 1962 et celui de 1968 une progression plus forte du pourcentage de salariés parmi les femmes (de 67,2\% en 1962 à 72,6\% en 1968) que parmi les hommes (de 72,5\% en 1962 à 75,4\% en 1968). Mais ce sont sans doute les inégalités liées aux différences dans les qualifications, au niveau du personnel ouvrier, qui suggèrent les remarques les plus importantes. Suivant une statistique récente tirée de l'enquête trimestrielle du ministère du Travail ${ }^{13}$ en octobre 1970 , la répartition de 1000 ouvriers et de 1000 ouvrières suivant la qualification s'établissait comme suit pour l'ensemble des activités industrielles.

TABLEAU 1

\begin{tabular}{crr}
\hline & Hommes & Femmes \\
& & \\
\hline$p^{3}$ a & 120 & 17 \\
$\mathrm{p}^{2}$ & 181 & 21 \\
$\mathrm{p}^{1}$ & 187 & 89 \\
O.S. $^{2}$ & 202 & 199 \\
O.S. $^{1}$ & 139 & 347 \\
Mancuvres spécialisés & 89 & 144 \\
Manœuvres ordinaires & 48 & 119 \\
Jeunes apprentis & 34 & 64 \\
Total : & 1000 & 1000
\end{tabular}

Source : Document ronéotypé distribué par la Division de la statistique du ministère du Travail.

a On peut dire, d'une manière générale que les qualifications de $\mathrm{p}^{3}, \mathrm{p}^{2}, \mathrm{p}^{1}$, demandent un apprentissage en école, que les qualifications de O.S. ${ }^{2}$ et de O.S. ${ }^{1}$ demandent une mise au courant dans l'atelier et, le plus souvent, l'acquisition d'un «coup de main», acquisitions dont la durée est rarement supérieure à un mois pour arriver à la cadence requise.

Ces différences suivant le sexe dans les niveaux de qualifications suggèrent déjà des différences dans les conditions de travail. Des enquêtes ont en ou-

13. L'enquête trimestrielle du ministère du Travail, adressée à un échantillon d'environ 50000 entreprises apporte régulièrement des données dans le domaine des effectifs, de la durée du travail, des taux de salaire horaire. Elle étudie, à des intervalles irréguliers, la répartition suivant la qualification du personnel de ces entreprises. Les données précédentes, dans ce domaine, remontaient à 1964 . 
tre montré que, à qualification égale, et particulièrement dans les postes d'O.S. ${ }^{14}$ où elles sont nombreuses, les femmes exécutent les travaux les plus parcellaires, les plus répétitifs, les plus sédentaires, plus souvent rémunérés au rendement que les travaux masculins correspondants. Des études permettent de se demander si l'évolution actuelle des techniques et plus particulièrement le développement de l'automatisation ne risquent pas d'aboutir à une accentuation de ces différences dans un contexte d'insuffisance et d'inadéquation des actions de formation professionnelle des femmes ${ }^{15}$. Dans le domaine des salaires, d'autre part, l'enquête du ministère du Travail citée plus haut enregistre, à qualification égale, l'existence de différences entre salaires masculins et féminins. On ne connaît pas encore les résultats d'une loi récente (Loi du 22 décembre 1972) concernant l'égalité des rémunérations féminines et masculines pour un travail de valeur égale.

Nous venons de tenter une énumération rapide des caractéristiques essentielles, dans la période actuelle, du travail féminin en France, caractéristiques à peine atténuées par une évolution lente qui se situe surtout au niveau des professions nécessitant des études longues où la situation des femmes marque quelques progrès. Ces traits caractéristiques du travail féminin peuvent avoir, sur le plan syndical, des conséquences diverses : la répartition inégale, suivant les secteurs, de la main-d'œuvre féminine a pu, dans le passé, inciter les femmes à abandonner aux mains des hommes les responsabilités syndicales; elle peut, au contraire, les arnener à s'affirmer davantage. On pourrait citer, dans la période actuelle, des secteurs à majorité féminine, dans l'alimentation, dans le textile ou dans certaines branches des métaux où les responsabilités syndicales dans les entreprises, longtemps laissées aux seuls hommes, sont maintenant assumées par les femmes et où les actions syndicales sont menées avec une large participation féminine.

La prédominance des femmes dans les postes de faible qualification mérite un examen particulier. On a, certes, fait remarquer depuis longtemps que les travailleurs les moins combatifs sont généralement les plus dénués de qualifications. Cette observation ne pourrait manquer de s'appliquer aux femmes. Pour celles qui n'ont pas reçu de formation professionnelle, qui n'ont donc pas conscience de posséder un métier, il peut y avoir des raisons supplémentaires d'être peu disposées à défendre leurs droits sur le plan professionnel. Cette remarque doit toutefois être sérieusement remise en question dans la période actuelle, et c'est là un point extrêmement important. Dans les luttes syndicales, dans les négociations entre salariés et employeurs, dans les interventions auprès du gouvernement, l'accent est actuellement mis sur les conditions de travail (horaires, salaires, cadences, environnement, mode de rémunération, sécurité de l'emploi). Or, il apparaît que la main-d'œuvre féminine est, avec la main-d'œuvre étrangère, celle dont les conditions de travail sont les plus souvent mises en cause. Il y a là un fait nouveau qui, d'une manière très nette, a déterminé, depuis quelques années, et particulièrement dans une période récente, un courant de réflexion dans toutes les instances et chez les travailleuses elles-mêmes. Des mouvements spontanés, des journées d'étude organisées par les syndicats mettent l'accent sur

14. M. Guilbert, les Fonctions des femmes dans l'industrie, Paris, Mouton, 1966, 394 p. 15. M. Guilbert, N. Lowit et J. Creusen, l'Évolution industrielle et le travail des femmes. Enquêti dans les industries des métaux, Centre d'études sociologiques, C.O.R.D.E., 1973, 96 p., document ronéotypé. 
les conditions de travail et sont, sans aucun doute, à l'origine d'un renforcement de la participation syndicale et de l'action syndicale parmi les femmes.

Il faut toutefois, ici, soulever un problème qui ne peut manquer de se poser aux organisations syndicales. S'il existe des conditions de travail particulièrement répandues parmi les femmes, sous quelle forme convient-il de présenter les revendications spécifiques qu'elles entraînent? Il semble bien que, dans les dernières années, ce problème soit l'un de ceux qui ont suscité les réflexions des centrales syndicales, tant au niveau de la formulation des revendications qu'au niveau de l'organisation de la propagande ou de la presse syndicale. Si les objectifs sont presque toujours communs, les solutions adoptées sont parfois différentes.

Le mouvement syndical français présente en effet plusieurs visages. La scission de la C.G.T. (Confédération générale du travail), en 1948, a donné naissance, à côté de l'ancienne C.G.T., à la C.G.T.-F.O. (Confédération générale du travail-Force ouvrière). La C.F.T.C. (Confédération française des travailleurs chrétiens), créée en 1920, s'est scindée en 1967 en deux organisations, la C.F.T.C. maintenue qui gardait un caractère confessionnèl et la C.F.D.T. (Confédération française des travailleurs). Quant à la C.G.C. (Confédération générale des cadres), elle regroupe un certain nombre de cadres, d'autres étant affiliés aux centrales ouvrières énumérées plus haut.

Il est difficile de connaître l'importance numérique des adhésions féminines à chaque centrale ou le pourcentage de syndiquées parmi les adhérents. Le décompte des femmes exerçant des responsabilités, s'il est possible au niveau des organismes centraux, est difficile au niveau des organismes de base où les changements sont fréquents. Pour tenter de recueillir quelques données concernant la période actuelle, nous nous sommes adressée aux différentes centrales syndicales, pour obtenir leurs chiffres ou leurs estimations dans ce domaine et définir les grandes lignes de leur politique concernant la main-d'œuvre féminine.

Pour les quatre centrales interrogées, les chiffres concernant l'affiliation sont approximatifs. La C.G.T. estime à environ $30 \%$ la proportion de femmes parmi ses 2300000 syndiqués; l'estimation en pourcentage est identique pour la C.F.D.T. La C.G.T.-F.O. fait remarquer qu'elle est bien implantée dans des secteurs à forte majorité féminine (fonctionnaires et secteurs tertiaires), la C.F.T.C. estime approximativement que les femmes représentent le tiers de ses effectifs. Ces pourcentages doivent évidemment être rapportés aux effectifs de chaque centrale qui sont en général mal connus ${ }^{16}$. On affirme globalement dans

16. À titre d'exemple voici d'après la Revue française des affaires sociales de juilletseptembre 1971, revue publiée par le ministère du Travail, la répartition en pourcentage des voix lors des élections, en 1970, des délégués aux comités d'entreprise. Les résultats concernent 7242 établissements. Ils ont été transmis par les inspecteurs du Travail. On sait que le premier collège groupe ouvriers et employés. Le second collège comprend généralement les techniciens, les dessinateurs, les agents de maîtrise, les cadres et ingénieurs.

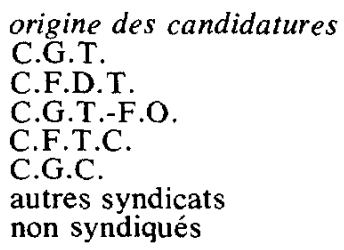

total

ler collège
53,9
20,2
7,4
2,6
$\overline{5}, 9$
10
100

$2 e$ collège
16,5
17,5
7,2
3,3
25,8
11,2
18,5

100

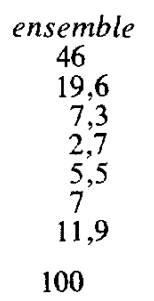


toutes les centrales syndicales que le taux de syndicalisation des femmes est actuellement sensiblement le même que celui des hommes ${ }^{17}$. Il peut toutefois varier suivant les secteurs.

L'accès aux responsabilités soulève d'autres problèmes. La C.G.T. signale que «cet accès est souhaité, suscité, encouragé de diverses manières. Dans l'entreprise, et notamment grâce à l'obtention de l'exercice des libertés syndicales ${ }^{18}$, les femmes accèdent facilement aux responsabilités de délégués du personnel, délégués au comité d'entreprise, collectrices, membres de la direction du syndicat d'entreprise. Leur nombre peut se chiffrer par plusieurs dizaines de milliers. Ces femmes assument leurs tâches syndicales avec beaucoup d'intelligence, de courage et d'esprit de responsabilité; se révélant souvent comme de véritables dirigeantes lors, notamment, des actions, grèves, etc. Cependant, à partir du moment où ces tâches se situent à un autre niveau, elles exigent d'être exercées en dehors du lieu et du temps de travail, elles nécessitent l'acquisition d'une formation syndicale plus élevée ce qui suppose de disposer de temps d'étude et de réflexion. Les choses deviennent alors plus difficiles».

D'une étude faite par la C.G.T. dans 15 de ses fédérations d'industrie il ressort que l'on trouve $17,9 \%$ de femmes parmi les membres des commissions exécutives, 18,2\% parmi les membres des bureaux, 13,8\% parmi les membres des secrétariats. Une étude auprès de 83 unions départementales fait apparaître que $12,9 \%$ des membres des bureaux sont des femmes, ainsi que $7,8 \%$ des membres des secrétariats. On relève en même temps que 24 femmes occupent des postes permanents de secrétaires d'unions départementales et que 28 femmes sont secrétaires permanentes de fédérations nationales. Parmi ces dernières, 3 sont secrétaires générales de leurs fédérations. Dans la C.G.T. encore, 16 femmes sont membres de la Commission exécutive confédérale qui compte 87 élus, deux femmes sont membres du Bureau confédéral, la plus haute instance de la C.G.T. qui compte 15 élus.

À la C.F.D.T., on explique que «les travailleuses prennent des responsabilités dans une proportion très différente suivant le niveau de la structure syndicale. La proportion de militantes (collectrices, déléguées du personnel) est importante tant qu'il s'agit d'une activité qui s'exerce dans l'entreprise. Il n'en est plus de même dès que l'on quitte ce niveau car, à ce moment, les responsabilités syndicales entraînent la participation à des réunions, en dehors du temps de travail. Les travailleuses mères de famille ou même simplement mariées rencontrent donc à ce moment des difficultés importantes pour concilier leur responsabilité familiale et une activité militante, d'où leur faible représentation dans les organismes statutaires». Une fédération nationale de la C.F.D.T. a une femme comme secrétaire confédérale. Sur 95 unions départementales, deux sont dirigées par des militantes. Au Conseil national confédéral il y a une dizaine de militantes sur 120 délégués. Le Bureau national compte deux membres féminins sur 31 et la Commission exécutive comprend une femme sur 10 responsables confédéraux.

17. La C.G.T. cite l'exemple d'une étude faite par sa Fédération des cuirs et peaux. Elle a porté sur 27 entreprises regroupant 3165 salariés dont 1170 femmes, soit $56 \%$ de l'effectif. On y trouve 917 femmes syndiquées sur 1725 adhérents de la C.G.T. soit 53\%.

18. Il s'agit de la loi du 27 décembre 1968 sur le droit syndical dans l'entreprise qui avait fait l'objet d'une annexe spéciale du protocole de Grenelle après les grèves de mai-juin 1968. 
À la C.G.T.-F.O., on estime que «un fait est à constater. L'intérêt des femmes pour le syndicalisme croît dans la mesure où elles sont attachées à leur vie professionnelle et où celle-ci leur apporte des satisfactions tant matérielles qu'intellectuelles [...] Intelligentes, sincères, dévouées, les femmes sont appréciées comme collectrices, déléguées du personnel ou de comités d'entreprise, secrétaires de syndicats, trésorières, membres de commissions exécutives. Très souvent, dans les petites entreprises notamment, la vie d'une section syndicale ou d'un syndicat repose sur une seule militante qui cumule les responsabilités. Mais à mesure que s'élève la promotion syndicale, la présence des femmes s'estompe. On les retrouve comme secrétaires d'unions locales, secrétaires administratives d'unions départementales ou de fédérations mais aucune n'a jamais été présentée à l'élection de secrétaire général d'union départementale. Il existe actuellement une secrétaire de fédération et il y a eu une femme au Bureau confédéral».

La C.F.T.C. estime que le pourcentage de femmes au niveau de la représentation dans les entreprises est assez important dans le secteur tertiaire mais assez difficile à préciser. Dans les organes statutaires où les statuts fixent le nombre des membres des conseils et des bureaux, «la participation féminine est assez largement minorée et ne correspond pas au nombre de femmes syndiquées dans les organisations intéressées [...] Dans les unions départementales, on trouve une représentation féminine en nombre variable. Pour les bureaux, dans une douzaine (sur une trentaine) on trouve une présidente, trois vice-présidentes, quatre secrétaires générales, quatre trésorières [...] Ce Conseil confédéral composé de 44 membres (dont 22 présentés par les organismes les plus importants et 22 élus par le congrès) comprend deux femmes présentées par leur organisation et une femme élue par le congrès (sur une liste de 30 candidats comprenant deux femmes) [...] Sur les trois femmes, membres du Conseil confédéral, une fait partie du Bureau».

On voit donc - et toutes les centrales le font remarquer - que les obstacles et les difficultés dont nous parlions plus haut jouent de moins en moins au niveau de l'entreprise où les femmes sont actives mais qu'ils se manifestent d'autant plus que le degré de responsabilité est le plus élevé. En ce qui concerne les revendications, les objectifs et les modes d'actions, nour reproduisons ci-dessus les passages essentiels des observations qui nous ont été communiquées.

La C.G.T., "considérant que la main-d'œuvre féminine figure parmi les plus exploitées et que la participation de plus du tiers des salariés que représentent les femmes est indispensable pour mener à bien ses objectifs revendicatifs, se préoccupe d'une façon particulière des revendications spécifiques des travailleuses [...] Depuis 1958, une Conférence nationale groupant les responsables au plus haut niveau (fédérations, unions départementales, syndicats) et des délégués hommes et femmes ( $1 / 3$ d'hommes, $2 / 3$ de femmes) se tient tous les quatre ans [...] La dernière s'est tenue les 17 et 18 mai 1973 et a rassemblé 2200 participants et participantes ${ }^{19}$ [...] La C.G.T. a également organisé en 1965 et 1967 des rassemblements unitaires concernant le temps de travail des femmes et l'égalité de salaire, de formation, de promotion ».

19. Cette conférence a donné lieu à une publication qui reproduit les principales interventions, les Femmes salariées, Paris, Éditions sociales, 1973, 247 p. 
La C.F.D.T. précise que «les revendications établies par la C.F.D.T. s'opposent à une politique menée par le gouvernement et le patronat pour inciter la femme à rester au foyer. La Confédération lutte pour que les travailleuses mères de famille aient à leur disposition les équipements collectifs nécessaires pour pouvoir choisir librement la forme de leur insertion sociale. Dans ce but, elle préconise également une modification des conditions d'attribution des prestations familiales et une augmentation importante de leur taux ».

À la C.G.T.-F.O., on nous dit «nous cherchons par tous les moyens, et avec le concours de militants persuadés du bien-fondé des revendications féminines, à créer des commissions chargées de l'étude des problèmes féminins, constituant des groupes de pression à tous les niveaux de l'organisation et obligeant les responsables syndicaux à examiner les problèmes dans une optique de mixité fondamentale».

À la C.F.T.C., on fait remarquer que «les revendications générales défendues par l'ensemble du mouvement concernent, bien entendu, les travailleurs et les travailleuses. Mais lorsqu'on entreprend une action pour les défavorisés, il est certain que les résultats seront surtout importants pour les femmes, si nombreuses dans ces catégories. Il en est ainsi de l'action entreprise concernant la revalorisation du S.M.I.G. ${ }^{20}$, des retraites de Sécurité sociale, de la retraite complémentaire, de la garantie de l'emploi, etc. Notre grande préoccupation est de persuader les femmes que leur sort dépend surtout d'elles-mêmes et que c'est dans la mesure où elles participeront à l'action syndicale [...] que la promotion féminine et, par là même, la promotion de toute la classe ouvrière aura des chances d'être réalisée».

Le problème de l'existence d'une presse syndicale féminine amène des remarques qu'il est intéressant de rapporter ici. À la C.F.D.T., on fait remarquer que «les travailleuses s'expriment dans la presse confédérale car la C.F.D.T. n'est pas favorable à une expression séparée des travailleuses qui conforterait la division traditionnelle des rôles entre hommes et femmes».

À la C.G.T.-F.O., on nous dit «nous n'avons jamais voulu de presse spécifiquement féminine. Quelle que soit la manière dont elle est présentée, cette presse reste forcément traditionnelle. Si certains de ces journaux font une plus large place aux problèmes sociaux, ils n'en maintiennent pas moins la conception du rôle spécifique de la femme au foyer».

La C.F.T.C. signale que, «dans l'organisme confédéral, Syndicalisme C.F.T.C., il n'y a pas une rubrique régulière pour traiter les problèmes du travail féminin. Cela tient en partie aux dimensions encore trop réduites de ce journal. Mais nous passons des informations d'actualité sur le sujet...». Quant à la C.G.T. qui possède, à côté de son hebdomadaire général, la Vie ouvrière, un mensuel féminin, Antoinette, elle s'exprime longuement sur ce problème et c'est pourquoi nous la citons ici en dernier lieu : "Pourquoi un journal fémirin alors que la C.G.T. lutte pour l'égalité des sexes dans le travail et dans la société? D'abord parce que les nombreuses inégalités qui subsistent nécessitent que toutes les travailleuses prennent conscience de cette situation

20. Salaire minimum interprofessionnel de croissance, précédemment désigné par le sigle S.M.I.G. (salaire minimum interprofessionnel garanti). 
infériorisée, connaissent leurs droits et les revendications particulières que suscite cette situation ainsi que les luttes qui sont menées pour leur aboutissement par telle ou telle catégorie de femmes en France ou à l'étranger. Ensuite parce que la vie des travailleuses ne s'arrête pas à la porte de l'entreprise. Les soucis du ménage, des enfants y entrent avec elles. Les aider à les résoudre, à partager ces tâches avec toute la famille, à lutter pour que des équipements collectifs, en ce qui concerne la garde et l'éducation des enfants et les travaux ménagers, allègent la double tâche que, pour l'instant, dans l'immense majorité des cas, elles assument seules est aussi l'objectif du journal. De même, en tenant compte du peu de temps dont elles disposent, des pages qui leur donnent des informations sur la vie culturelle leur permettent d'accéder à l'idée que, dans une société plus juste, moins contraignante, d'autres horizons pourraient leur être ouverts. Le journal féminin Antoinette a aussi pris l'initiative de l'organisation de colloques, l'un en novembre 1970 sur le thème «Le droit au travail des femmes est-il menacé? Les femmes vont-elles manquer la révolution industrielle?», l'autre en janvier 1973 abordant un autre sujet d'actualité : «Les conditions de travail des femmes. Vérités et solutions». $\grave{A}$ ces deux colloques ont participé, outre des syndicalistes hommes et femmes, des sociologues, des économistes, des ergonomes, des médecins. »

Nous n'ajouterons que quelques remarques. Elles confirment ce que nous avions dit précédemment concernant des progrès accomplis dans la participation syndicale des femmes. Elles mettent aussi en lumière des difficultés que nous avions soulignées. Elles expriment des convergences fondamentales sur le plan des revendications en même temps qu'elles font apparaître certaines divergences entre les centrales syndicales concernant les modes d'action. Peut-être ces dernières pages laissent-elles au lecteur l'impression d'une situation encore encombrée d'obstacles. Il conviendrait alors, avant de terminer, d'insister sur ce que ces témoignages nous ont appris quant à l'importance de la participation des femmes sur le lieu de travail. Peut-être faudrait-il aussi rappeler ce que nous avons dit plus haut concernant la sensibilisation croissante des femmes à leurs conditions de travail. La presse nous apporte chaque jour les échos d'actions menées dans l'habillement, les industries mécaniques, les industries chimiques notamment, dans un certain nombre d'entreprises employant une majorité de femmes, où elles ont pris la tête de mouvements qui les concernent au premier chef, pour l'amélioration des salaires, des conditions de travail, contre les licenciements. En fait, tous les aspects que nous avons décrits coexistent et nous souhaitons que l'on ait pu déceler, dans l'exposé que nous avons tenté d'en faire, la complexité d'une situation qui semble être entrée, dans la période actuelle, dans une phase d'évolution rapide.

\section{RÉSUMÉ}

Pour aborder les problèmes des rapports entre les femmes et les syndicats en France il faut d'abord replacer les faits dans leur contexte historique. La montée de la main-d'œuvre féminine, mise en situation de concurrence avec la main-d'œuvre masculine, a suscité, dès la seconde moitié du 19. siècle, dans le mouvement syndical, des inquiétudes et des oppositions qui n'ont été surmontées que lentement. Si on examine les faits actuels, il faut tenir compte d'une situation 
particulière des femmes, sur le plan familial comme sur le plan du travail. Les taux d'activité des femmes sont, depuis plusieurs années, en nette augmentation mais la répartition des femmes actives, notamment suivant la catégorie socio-professionnelle ou la qualification, est différente de celle des hommes. Ces faits peuvent avoir des conséquences négatives. En même temps cependant l'attention actuellement portée aux problèmes des conditions de travail ne peut manquer d'être favorable au développement de la participation des femmes aux luttes ouvrières. La pluralité syndicale en France rend difficile une appréciation globale de l'état actuel de la participation syndicale des femmes. Importante à la base, celle-ci est moins importante à mesure que l'on s'élève dans l'échelle des responsabilités. Il est certain toutefois que la période actuelle est marquée par une accentuation de la participation et de l'initiative des femmes dans les actions syndicales.

\begin{abstract}
[Women and Unions in France] In order to confront problems in the relationship between women and unions in France, one must first place the facts in historical context. The increase in women workers, implying competition with working men, created, as early as the second half of the 19th century, anxieties and resistance that were only slowly overcome. When looking at present facts, one must take into consideration the particular situation of women, in the family as well as in the work world. Women's activities have been clearly increasing for several years, but the distribution of active women, especially in terms of socio-professional categories or in terms of qualifications, is different from that of men. These facts may have negative consequences. Nevertheless at present the attention spent on working conditions can only be favorable to the increase of women's participation in working class struggles. The plurality of unions in France makes an over all appreciation of the p:esent state of women's union participation difficult. Important at the grass roots, this is less important as one rises in the hierarchy of responsabilities. It is nonetheless certain that at present women's participation and initiative in union action is increasing.
\end{abstract}

\title{
RESUMEN
}

[Mujeres y sindicatos en Francia] Para abordar los problemas de las relaciones entre las mujeres y los sindicatos en Francia es necesario primeramente remplazar los hechos en su contexto histórico. La augmentación de la mano de obra femenina, puesta en situación de concurrencia con la mano de obra masculina, a creado desde la segunda mitad del siglo 19 , en el movimiento sindical, inquietudes y oposiciones que no han sido sobrepasadas que lentamente. Si se examina los hechos actuales, hay que tener en cuenta de una situación particular de las mujeres tanto en el plano familiar como en el plano del trabajo. Los porcentages de actividad de las mujeres, son despues de muchos años en neta augmentación pero la repartición de la mujeres activas, principalmente siguiendo las categorias socio-profecionales o las calificaciones, es diferente de aquella de los hombres. Esos hechos pueden tener consecuencias negativas. Al mismo tiempo, sin embargo, la atención actualmente prestada a los problemas de las condiciones de trabajo no pueden fallar de ser favorables al desarrollo de la participación de las mujeres a las luchas obreras. La pluralidad sindical en Francia hace dificil una apreciación global del estado actual de la participación sindical de las mujeres. Importante a la base, ella es menos importante a medida que se eleva en la escala de responsabilidades. Es seguro, no obstante que el periodo actual está marcado por una accentuación de la participación y de la iniciativa de las mujeres en las acciones sindicales. 